\title{
Free Energy and Phase Transition of the Matrix Model on a Plane-Wave
}

\author{
Shirin Hadizadeh, Bojan Ramadanovic, \\ Gordon W. Semenoff and Donovan Young \\ Department of Physics and Astronomy, \\ University of British Columbia, \\ 6224 Agricultural Road, \\ Vancouver, British Columbia, Canada V6T 1Z1 \\ shirin, bramadan, dyoung@physics.ubc.ca semenoff@nbi.dk
}

June 24, 2021

\begin{abstract}
It has recently been observed that the weakly coupled plane wave matrix model has a density of states which grows exponentially at high energy. This implies that the model has a phase transition. The transition appears to be of first order. However, its exact nature is sensitive to interactions. In this paper, we analyze the effect of interactions by computing the relevant parts of the effective potential for the Polyakov loop operator in the finite temperature plane-wave matrix model to three loop order. We show that the phase transition is indeed of first order. We also compute the correction to the Hagedorn temperature to order two loops.
\end{abstract}




\section{Introduction}

It is conjectured that the full dynamics of M-theory is encoded in a certain maximally supersymmetric matrix quantum mechanics called the BFSS matrix model [1. At finite $N$ that model is thought to describe a discrete light-cone quantization of M-theory in the infinite momentum frame. The light-cone is decompactified by a taking a particular large $N$ limit where the discrete lightcone momentum $p^{+}=N / R$ is held constant, with $R$ the radius of the null identification $x^{-} \sim x^{-}+2 \pi R$.

Recently, a massive deformation of the BFSS matrix model appropriate to M-theory on a plane wave background has been studied [2]. The main difference between it and the BFSS model is their supersymmetry, which in the latter case is that of a maximally supersymmetric plane wave spacetime rather than 11-dimensional Minkowski space. A great advantage of the plane wave matrix model is that it has a weak coupling regime where it can be studied systematically using perturbation theory 3 - 11. It also has a powerful supersymmetry algebra which allows the extrapolation of some perturbative results to the string coupling regime.

The action of the plane wave matrix model is

$$
\begin{aligned}
S=\frac{1}{2 R} \int d \tau \operatorname{Tr}\left(D X^{i} D X^{i}+D X^{\bar{a}} D X^{\bar{a}}+i \psi^{\dagger I \alpha} D \psi_{I \alpha}+\frac{R^{2}}{2}\left[X^{i}, X^{j}\right]^{2}+\right. \\
+\frac{R^{2}}{2}\left[X^{\bar{a}}, X^{\bar{b}}\right]^{2}+R^{2}\left[X^{\bar{a}}, X^{i}\right]^{2}+\frac{R^{2}}{2}\left[X^{i}, X^{j}\right]^{2}+\frac{R^{2}}{2}\left[X^{\bar{a}}, X^{\bar{b}}\right]^{2}+R^{2}\left[X^{\bar{a}}, X^{i}\right]^{2} \\
-R \psi^{\dagger I \alpha} \sigma_{\alpha}^{\bar{a} \beta}\left[X^{\bar{a}}, \psi_{I \beta}\right]+\frac{R}{2} \epsilon_{\alpha \beta} \psi^{\dagger \alpha I} g_{I J}^{i}\left[X^{i}, \psi^{\dagger \beta J}\right]-\frac{R}{2} \epsilon^{\alpha \beta} \psi_{\alpha I}\left(g^{i \dagger}\right)^{I J}\left[X^{i}, \psi_{\alpha J}\right] \\
\left.-\left(\frac{\mu}{3}\right)^{2}\left(X^{\bar{a}}\right)^{2}-\left(\frac{\mu}{6}\right)^{2}\left(X^{i}\right)^{2}-\frac{\mu}{4} \psi^{\dagger I \alpha} \psi_{I \alpha}-i \mu \frac{2 R}{3} \epsilon_{\bar{a} \bar{b} \bar{c}} X^{\bar{a}} X^{\bar{b}} X^{\bar{c}}\right)
\end{aligned}
$$

where all of the variables are $N \times N$ matrices. The indices on the bosonic matrices take values $\bar{a}, \bar{b}, \bar{c}=1,2,3, i, j=4, \ldots, 9$. The $\mathrm{U}(\mathrm{N})$ symmetry is gauged. All variables transform in the adjoint representation of the gauge group $X^{i} \rightarrow U X^{i} U^{\dagger}$, etc. The time derivatives are covariant, $D=\partial_{\tau}-i[A, \ldots]$ with an $N \times N$ Hermitian gauge field $A$. The fermions have 8 complex components with $I, J=1, \ldots, 4$ and $\alpha, \beta=1,2$. The spin matrix has the property $\mathrm{g}^{i}\left(\mathrm{~g}^{j}\right)^{\dagger}+$ $\mathrm{g}^{j}\left(\mathrm{~g}^{i}\right)^{\dagger}=2 \delta^{i j} \mathbf{1}_{4 \times 4} . \epsilon_{\alpha \beta}$ and $\epsilon_{\bar{a} \bar{b} \bar{c}}$ are antisymmetric tensors.

Note that the last line of (11) contains terms with the parameter $\mu$ which comes from the plane wave geometry. That geometry has metric and constant four-form,

$$
d s^{2}=-2 d x^{+} d x^{-}+d x^{a} d x^{a}+d x^{i} d x^{i}-d x^{+} d x^{+}\left(\left(\frac{\mu}{3}\right)^{2}\left(x^{a}\right)^{2}+\left(\frac{\mu}{6}\right)^{2}\left(x^{i}\right)^{2}\right)
$$




$$
F_{123+}=\mu
$$

It is a maximally symmetric solution of 11-dimensional supergravity for any value of the parameter $\mu$. If we set $\mu$ to zero we recover the Minkowski metric.

The plane wave matrix model (10) can be systematically analyzed in perturbation theory. When $N$ is large, the expansion parameter is [12]

$$
\lambda=\left(\frac{3 R}{\mu}\right)^{3} N
$$

Note that all dimensional quantities are in units of the eleven dimensional Planck length which we have set to one.

Aside from being a model of M-theory on a plane-wave background, there is an interesting connection between the matrix model (11) and four dimensional $\mathcal{N}=4$ supersymmetric Yang-Mills theory. At the classical level, the degrees freedom and dynamics described by (1) are a consistent truncation of the YangMills theory which keeps only those modes which are invariant under a certain $S U(2)$ subalgebra of the full $S U(2,2 \mid 4)$ superconformal algebra. In the planar limit, this truncation seems to also hold at the one-loop level [9]. It is then natural to speculate about whether it would inherit some of the properties of $\mathcal{N}=4$ supersymmetric Yang-Mills theory, such as integrability of the large $N$ limit, at least in the leading orders of perturbation theory. This has been studied in detail in recent work [1]. It has been shown that the form of the dilatation operator for supersymmetric Yang-Mills theory which has been conjectured using integrability as an input, when restricted to the appropriate sector of the theory, is identical to the Hamiltonian of the plane wave matrix model, at least for computing energy levels to three loop order.

\section{$1.1 \quad$ Thermodynamics}

Some aspects of the thermodynamics of the plane wave matrix model have been studied before [13-[18. It was shown in 13] that, at weak coupling, and in the large $\mathrm{N}$ limit, there is a deconfinement phase transition at a particular temperature. The use of the word "deconfinement" in a theory where there is no spatial extent over which particles can be separated must be justified carefully. It is possible to distinguish the two phases by the behavior of the free energy, $F[T]$. In a weakly coupled theory, the free energy should be proportional to the number of degrees of freedom. In the confining phase the number of degrees of freedom at a given energy are of order the number of color singlets, which in the lower energy parts of the spectrum does not grow with the rank of the

gauge group, $N$, but is of order one. In a deconfined phase, the number of 
degrees of freedom is the number of elements of the matrices, which is of order $N^{2}$. Thus, we would expect

$$
\begin{aligned}
& \lim _{N \rightarrow \infty} \frac{F}{N^{2}}=0 \text { confined } \\
& \lim _{N \rightarrow \infty} \frac{F}{N^{2}} \neq 0 \text { deconfined }
\end{aligned}
$$

When interactions are turned on, the large $N$ limit which we are using is the 't Hooft limit [12, where the coupling constants are also scaled as $N$ is taken large so that $\lambda$ defined in (2) is held constant. Note that this limit is different from the large $N$ limit with $N / R$ held constant which would decompactify the null direction in M-theory.

In a gauge theory such as (11), where all variables transform in the adjoint representation, there is an order parameter for confinement called the Polyakov loop [19, 20]. It is the trace of the holonomy of the gauge field around the finite temperature Euclidean time circle,

$$
P=\frac{1}{N} \operatorname{Tr}\left(e^{i \oint A}\right)
$$

It is related to the difference of free energies of the state where an additional fundamental representation quark is introduced and the state without the quark,

$$
F_{q}[T]-F_{0}[T]=-T \ln \langle P\rangle
$$

The free energy of a free quark in the confined phase should be large compared to that in the deconfined phase. In fact,

$$
\begin{array}{ccc}
\langle P\rangle & =0 & \text { confined } \\
\langle P\rangle & \neq 0 & \text { deconfined }
\end{array}
$$

is interpreted as requiring infinite energy to insert a fundamental representation quark into the system when it is in the confining phase, whereas it is finite in the deconfined phase. The Polyakov loop operator is widely used to study finite temperature de-confining phase transitions in higher dimensional gauge theories, 21]-30]

A phase transition where both criteria for deconfinement (3) and (5) occur has been found in the large $N$ limit of the matrix model [13. It has been argued to be generic to the large $N$ limit of matrix quantum mechanics when the matrices have a gapped spectrum. The transition temperature is of order of the mass gap.

An intuition for why this phase transition occurs can be gained from counting matrix degrees of freedom [13]. As a simple example, consider a set of $d$ 
matrix harmonic oscillators with Hamiltonian

$$
H=\sum_{i=1}^{d} \omega \operatorname{Tr}\left(\alpha_{i}^{\dagger} \alpha_{i}\right)
$$

and matrix-valued creation and annihilation operators with algebra

$$
\left[\alpha_{a b}^{i}, \alpha_{c d}^{j \dagger}\right]=\delta^{i j} \delta_{a d} \delta_{b c}
$$

States are created the creation operators $\alpha_{a b}^{i \dagger}$ operating on a vacuum $\mid 0>$. To get a state with energy $E=n \omega$ we must act with $n$ creation operators.

The analog of gauge symmetry is to require a physical state condition of invariance under the unitary transformation

$$
\alpha^{\dagger} \rightarrow u \alpha^{\dagger} u^{\dagger} \quad, \quad \alpha \rightarrow u \alpha u^{\dagger}
$$

where $u \in U(N)$. We assume that the vacuum state is invariant. Then, physical states are created by operating with invariant combinations of creation operators. In the limit $N \rightarrow \infty$ all such combinations are traces

$$
\left[\operatorname{Tr}\left(\alpha_{i}^{\dagger}\right)\right]^{n_{1}}\left[\operatorname{Tr}\left(\alpha_{j_{1}}^{\dagger} \alpha_{j_{2}}^{\dagger}\right)\right]^{n_{2}}\left[\operatorname{Tr}\left(\alpha_{k_{1}}^{\dagger} \alpha_{k_{2}}^{\dagger} \alpha_{k_{3}}^{\dagger}\right)\right]^{n_{3}} \ldots \mid 0>
$$

where the energy is

$$
E=\omega\left(n_{1}+2 n_{2}+3 n_{3}+\ldots\right)
$$

The number of these traces with a fixed energy, $E$, does not scale like $N^{2}$ as $N \rightarrow \infty$, instead it approaches a constant as $N$ is taken large. Thus at low enough temperatures, the free energy should approach an $N$-independent constant as $N$ is taken large.

However, the number of independent traces does increase rapidly with the energy $E$. It has been shown [13] that, in the large $N$ limit, the oscillator has a Hagedorn density of states at high energy,

$$
\rho(E) \sim \frac{1}{E} e^{E / T_{H}}
$$

where the Hagedorn temperature is

$$
T_{H}=\omega / \ln d
$$

This mirrors a similar discussion for weakly coupled Yang-Mills theory 31-36].

At temperatures higher than $T_{H}$, the thermodynamic canonical ensemble does not exist. It could be made to exist by keeping $N$ large but finite. That would cut off the exponential growth in the asymptotic density of states at some large energy, of order $N^{2}$. Then we could consider a temperature that is 
greater than $T_{H}$. Both the energy and entropy would be dominated by states at and above the cutoff scale and would be of order $N^{2}$. The divergence of the free energy $\sim N^{2}$ occurs as we take the limit $N \rightarrow \infty$ at constant temperature (noting that the Hagedorn temperature does not depend on $N$ ).

It was shown in ref. 13 that, from a different point of view, this behavior of the matrix model can also be found as a large $N$ Gross-Witten type of phase transition [37, 38, which is familiar in unitary matrix models. In the next Section, we will discuss this in more detail.

\section{One loop}

Now, let us consider a perturbative expansion of the plane wave matrix model at finite temperature. The partition function has Euclidean path integral representation similar to those of finite temperature quantum field theories. We consider the theory in Euclidean space where time is compact and identified as

$$
\tau \sim \tau+\beta
$$

with

$$
\beta=1 / T
$$

$T$ is the temperature. The partition function is given by the functional integral

$$
Z=\int[d A]\left[d X^{i}\right][d \psi] e^{-\int_{0}^{\beta} d \tau L\left[A, X^{i}, \psi\right]}
$$

where $L$ is the Euclidean time Lagrangian

$$
\begin{aligned}
& L=\frac{1}{2 R} \operatorname{Tr}\left(D X^{i} D X^{i}+D X^{\bar{a}} D X^{\bar{a}}-\psi^{\dagger I \alpha} D \psi_{I \alpha}\right) \\
& +\frac{1}{2 R} \operatorname{Tr}\left(\left(\frac{\mu}{3}\right)^{2}\left(X^{\bar{a}}\right)^{2}+\left(\frac{\mu}{6}\right)^{2}\left(X^{i}\right)^{2}+\frac{\mu}{4} \psi^{\dagger I \alpha} \psi_{I \alpha}+i \mu \frac{2 R}{3} \epsilon_{\bar{a} \bar{b} \bar{c}} X^{\bar{a}} X^{\bar{b}} X^{\bar{c}}\right. \\
& \quad+R \psi^{\dagger I \alpha} \sigma_{\alpha}^{\bar{a} \beta}\left[X^{\bar{a}}, \psi_{I \beta}\right]-\frac{R}{2} \epsilon_{\alpha \beta} \psi^{\dagger \alpha I} \mathrm{~g}_{I J}^{i}\left[X^{i}, \psi^{\dagger \beta J}\right]+\frac{R}{2} \epsilon^{\alpha \beta} \psi_{\alpha I}\left(g^{i \dagger}\right)^{I J}\left[X^{i}, \psi_{\alpha J}\right] \\
& \left.\quad-\frac{R^{2}}{2}\left[X^{i}, X^{j}\right]^{2}-\frac{R^{2}}{2}\left[X^{\bar{a}}, X^{\bar{b}}\right]^{2}-R^{2}\left[X^{\bar{a}}, X^{i}\right]^{2}\right)
\end{aligned}
$$

The bosonic and fermionic variables have periodic and antiperiodic boundary conditions, respectively

$$
A(\tau+\beta)=A(\tau), \quad X^{i}(\tau+\beta)=X^{i}(\tau), \quad \psi(\tau+\beta)=-\psi(\tau)
$$

Since the boundary conditions for fermions and bosons are different, supersymmetry is broken explicitly. Of course this is expected at finite temperature 
where bosons and fermions have different thermal distributions. Supersymmetry is restored in the zero temperature limit. We will see the results of this explicitly in the following. A parallel discussion of the BFSS matrix model at finite temperature can be found in ref. [39]-40].

\subsection{Gauge fixing}

To begin, we must fix the gauge. It is most convenient to use the gauge freedom to make the variable $A$ static and diagonal,

$$
\frac{d}{d \tau} A_{a b}=0 \quad, \quad A_{a b}=A_{a} \delta_{a b}
$$

Once this is done, the remaining degrees of freedom of $A$ are the time-independent diagonal components, $A_{a}$. We shall see that they eventually appear in the form $\exp \left(i \beta A_{a}\right)$.

The Faddeev-Popov determinant for the first of these gauge fixings is ${ }^{1}$

$$
\operatorname{det}^{\prime}\left(-\frac{d}{d \tau}\left(-\frac{d}{d \tau}+i\left(A_{a}-A_{b}\right)\right)\right)=\operatorname{det}^{\prime}\left(-\frac{d}{d \tau}\right) \operatorname{det}^{\prime}\left(-\frac{d}{d \tau}+i\left(A_{a}-A_{b}\right)\right)
$$

where the boundary conditions are periodic with period $\beta$. The prime means that the zero mode of time derivative operating on periodic functions is omitted from the determinant. Once the gauge field is time-independent, we do the further gauge fixing which makes it diagonal. The Faddeev-Popov determinant for diagonalizing it is the familiar Vandermonde determinant,

$$
\prod_{a \neq b}\left|A_{a}-A_{b}\right|
$$

This is also just the factor that the time independent zero mode would contribute to the second of the determinants in (12). Including it gives the determinant

$$
\prod_{a \neq b} \operatorname{det}^{\prime}\left(-\frac{d}{d \tau}\right) \operatorname{det}\left(-\frac{d}{d \tau}+i\left(A_{a}-A_{b}\right)\right)
$$

where there is now no prime on the second factor. These determinants can be found explicitly. We will do this shortly.

\footnotetext{
${ }^{1}$ Using zeta-function regularization,

$$
\operatorname{det}^{\prime}\left(-\frac{d}{d \tau}\right)=\beta
$$
}




\section{$2.2 \quad$ Classical ground states}

We will perform a semiclassical expansion of the free energy in the sector of the theory with the classical ground state $X^{a}=0=X^{i}$ in the large $N$, 't Hooft limit. This is a double expansion. First, it keeps only planar Feynman diagrams, which is the leading order in an expansion in $1 / N^{2}$. Secondly, it is perturbative in that it keeps those diagrams which are of low orders in the coupling constant. Since $N$ is large, the appropriate coupling constant is $\lambda$ defined in eqn. (2).

The configuration $X^{a}=0=X^{i}$ has zero classical energy. In fact, because of supersymmetry, the ground state energy of the theory quantized beginning with this vacuum is zero to all orders in perturbation theory. This will provide us with an important check of our finite temperature computations. Of course, temperature breaks supersymmetry and the free energy of the thermodynamic state, which is what we compute, is non-zero. However, we will always be able to check whether the zero temperature limit of the free energy, which is the ground state energy, vanishes. We find that its expansion in the coupling constant $\lambda$ indeed does so to order $\lambda^{2}$.

In this subsection, for completeness, we comment on the fact that there are a large number $(\rightarrow \infty$ as $N \rightarrow \infty)$ of other ground states with zero energy. To see this, observe that the classical potential in (10) can be written in the form

$$
\begin{array}{r}
V=\frac{R}{2} \operatorname{Tr}\left[\left(\frac{\mu}{3 R} X^{a}+i \epsilon^{a b c} X^{b} X^{c}\right)^{2}+\frac{1}{2}\left(i\left[X^{i}, X^{j}\right]\right)^{2}+\right. \\
\left.+\left(i\left[X^{i}, X^{a}\right]\right)^{2}+\left(\frac{\mu}{6 R}\right)^{2}\left(X^{i}\right)^{2}\right]
\end{array}
$$

It has isolated global classical minima $(V=0)$ where

$$
X_{\mathrm{cl}}^{i}=0, \quad X_{\mathrm{cl}}^{a}=\frac{\mu}{3 R} J^{a}
$$

and $J^{a}$ form an N-dimensional representation of the $\mathrm{SU}(2)$ algebra, $\left[J^{a}, J^{b}\right]=$ $i \epsilon^{a b c} J^{c}$. In addition, the classical solution for gauge field must obey the equation

$$
\left[A_{\mathrm{cl}}, J_{\mathrm{cl}}^{a}\right]=0
$$

If $J^{a}$ are an irreducible representation of $\mathrm{SU}(2)$, by Schur's Lemma, $A_{\mathrm{cl}}$ must be proportional to the unit matrix $A_{\mathrm{cl}}=c \cdot \mathcal{I}$ and a symmetry of the theory allows us to set $c=0$. The gauge symmetry is realized by the Higgs mechanism and fluctuations of the gauge field are all massive. On the other hand, when the representation is reducible, there are gauge fields which commute with the condensate. The parts of $A_{\mathrm{cl}}$ which commute with the condensate 
remain undetermined by the classical equations. The volume of the space of all possible such $A_{\mathrm{cl}}$ forms a moduli space of the classical solutions which must still be integrated over, even to obtain the leading order in the semi-classical approximation to the partition function.

The implications of the solutions (15) have been discussed in detail in ref. 8 . They were interpreted in terms of the spherical membrane and transverse spherical 5-branes which exist on the 11-dimensional plane wave background.

In refs. 13, 17] it was argued that the phase transition that we shall study here occurs only when there is a residual gauge symmetry and only in the limit where the rank of the residual gauge group goes to infinity. According to ref. [8, this is the limit of the theory which describes 5-branes. Perturbation theory in that limit is governed by a 't Hooft coupling similar to (2) with $N$ replaced by the rank of the residual gauge group. Ref. 8 argued that, in the 't Hooft limit, the barrier between the degenerate vacua becomes infinitely high. They also argued that the limit decouples the 5-brane from other degrees of freedom and focuses on its internal dynamics.

The results of refs. [13, 17] can be interpreted as saying that the phase transition of the matrix model occurs only in 5-brane states and not in membrane states, and it seems to be associated with internal dynamics of the 5-branes. Of course, refs. [13, 17] analyzed only the weak coupling limit of the matrix model which is far from the limits which are conjectured to describe supergravity of an 11-dimensional spacetime continuum where the 5-brane would live. In order to apply it directly to any known behavior of 5-branes, it would have to be extrapolated to strong 't Hooft coupling. This is of course a difficult problem. The perturbative expansion in the present paper perhaps gives an indication that the phase transition is of first order but much more would have to be done to answer the question of persistence or nature of the phase transition in the 5-brane regime. One interesting extension of the present work would be to examine the dependence of the nature of the phase transition on the number of 5-branes. For $k$ coincident 5 -branes, $X^{a}$ contains $N / k k$-dimensional representations of $\mathrm{SU}(2)$, with $\mathrm{k}$ being held fixed in the large $\mathrm{N}$ limit. $k$-dependence of the phase transition temperature was investigated in ref. [13].

\subsection{Semiclassical expansion}

If we expand about the classical vacuum $X_{\mathrm{cl}}^{a}=0=X_{\mathrm{cl}}^{i}$, we find the partition function in the 1-loop approximation is

$$
Z=\int d A_{a} \prod_{a \neq b} \frac{\operatorname{det}^{\prime}(-d / d \tau) \operatorname{det}\left(-D_{a b}\right) \operatorname{det}^{8}\left(-D_{a b}+\frac{\mu}{4}\right)}{\operatorname{det}^{3 / 2}\left(-D_{a b}^{2}+\frac{\mu^{2}}{9}\right) \operatorname{det}^{3}\left(-D_{a b}^{2}+\frac{\mu^{2}}{36}\right)}
$$


where $D_{a b}=\frac{d}{d \tau}-i\left(A_{a}-A_{b}\right)$. The first two terms in the numerator are the Faddeev-Popov determinant. The third term comes from fermions whereas the denominator is from bosons. Using the formula

$$
\operatorname{det}\left(-\frac{d}{d \tau}+\omega\right)=2 \sinh \frac{\beta \omega}{2}
$$

with periodic boundary conditions and

$$
\operatorname{det}\left(-\frac{d}{d \tau}+\omega\right)=2 \cosh \frac{\beta \omega}{2}
$$

with antiperiodic boundary conditions, we can write ${ }^{2}$

$$
Z=\int_{-\pi}^{\pi} \prod_{a=1}^{N} \frac{d\left(\beta A_{a}\right)}{2 \pi} \prod_{a \neq b} \frac{\left[1-e^{i \beta\left(A_{a}-A_{b}\right)}\right]\left[1+e^{-\beta \mu / 4+i \beta\left(A_{a}-A_{b}\right)}\right]^{8}}{\left[1-e^{-\beta \mu / 3+i \beta\left(A_{a}-A_{b}\right)}\right]^{3}\left[1-e^{-\beta \mu / 6+i \beta\left(A_{a}-A_{b}\right)}\right]^{6}}
$$

Note that, because of supersymmetry, the zero temperature $(\beta \rightarrow \infty)$ limit of the partition function is one. It also has a symmetry under replacing $e^{-\beta \mu}$ by $1 / e^{-\beta \mu}$.

We must now do the remaining integral when $N \rightarrow \infty$. There are $N$ integration variables $A_{a}$ and the action, which is the logarithm of the integrand is generically of order $N^{2}$ which is large in the large $N$ limit. For this reason, the integral can be done by saddle point integration. This amounts to finding the configuration of the variables $A_{a}$ which minimize the effective action:

$$
\begin{aligned}
S_{\text {eff }} & =\sum_{a \neq b}\left(-\ln \left[1-e^{i \beta\left(A_{a}-A_{b}\right)}\right]-8 \ln \left[1+e^{-\beta \mu / 4+i \beta\left(A_{a}-A_{b}\right)}\right]+\right. \\
& \left.+3 \ln \left[1-e^{-\beta \mu / 3+i \beta\left(A_{a}-A_{b}\right)}\right]+6 \ln \left[1-e^{-\beta \mu / 6+i \beta\left(A_{a}-A_{b}\right)}\right]\right)
\end{aligned}
$$

To study the minima, it is illuminating to Taylor expand the logarithms in the phases (this requires some assumptions of convergence for the first log)

$$
S_{\mathrm{eff}}=\sum_{n=1}^{\infty} \frac{1-8(-)^{n+1} r^{3 n}-3 r^{4 n}-6 r^{2 n}}{n} \phi_{-n} \phi_{n}
$$

Here,

$$
r=\exp (-\beta \mu / 12)
$$

and

$$
\phi_{n}=\frac{1}{N} \sum_{a=1}^{N} e^{i n \beta A_{a}}
$$

\footnotetext{
${ }^{2}$ Because the matrix model action (1) is invariant under replacing $A$ by $A$ plus a constant times the unit matrix, we see that the integrand in eqn.(17) is indeed invariant under translating all values of $A_{a}$ by the same constant.
} 
Recalling (4), we note that $\phi_{n}$ are multiply wound Polyakov loop operators evaluated in the static, diagonal gauge. The zeroth moment is normalized

$$
\phi_{0}=1
$$

The other elements are constrained by sum rules. The density defined by

$$
\begin{aligned}
\rho(\chi) & =\frac{1}{N} \sum_{a=1}^{\infty} \delta\left(\chi-\beta A_{a}\right) \geq 0 \\
& =\sum_{n} e^{-2 \pi i n \chi} \phi_{n}
\end{aligned}
$$

is a non-negative function. For example, if only $\phi_{0}$ and $\phi_{ \pm 1}$ are nonzero, (22) implies that $\left|\phi_{1}\right| \leq 1 / 2$.

In this one-loop approximation, the action is quadratic in the Polyakov loops. When all coefficients of the quadratic terms are positive, the action is minimized by $\phi_{n}=0$ for $n \neq 0$. This is the confining phase. When a coefficient becomes negative, the effective action is minimized with one of the loops nonzero. The result is a condensation of the loops.

As we raise the temperature from zero (and lower $\beta$ from infinity), the first mode to condense is $n=1$. This occurs when

$$
r_{C}=1 / 3 \rightarrow T_{C}=\frac{\mu}{12 \ln 3} \approx .0758533 \mu
$$

and $\phi_{1} \neq 0$ when $T>T_{C}$. There are some speculations about the nature of the distribution of the angles $\beta A_{a}$ in the deconfined phase in ref. [17.

Note that this condensation breaks a $U(1)$ symmetry. This is associated with the center of the gauge group $U(1) \in U(N)$. It arises from the fact that all variables are in the adjoint representation. In the Euclidean path integral, gauge transformations $X(\tau) \rightarrow U(\tau) X(\tau) U^{\dagger}(\tau)$ must preserve the periodicity of the dynamical variables. They therefore must be periodic up to an element of the center, $U(\beta)=e^{i \theta} U(0)$. The Polyakov loop, on the other hand, being the holonomy on the time circle, does transform as $P \rightarrow e^{i \theta} P$.

Even once the static, diagonal gauge is fixed, there is a vestige of this symmetry where $\beta A_{a} \rightarrow \beta A_{a}+\theta$ or $\phi_{n} \rightarrow e^{i n \theta} \phi_{n}$. This symmetry restricts the form of the effective action for Polyakov loops, so that the term with $\phi_{k_{1}} \ldots \phi_{k_{n}}$ must have $\sum k_{i}=0$. It is a good symmetry of the confined phase and it is spontaneously broken in the deconfined phase. The Polyakov loop operator is an order parameter for this symmetry breaking. 


\section{$3 \quad$ Higher loop order}

In the previous Section, we have computed the effective action as a function of the variables $\phi_{n}$ in the one-loop approximation. The result is the quadratic potential in eqn. (19). To find the stable phase, it is necessary to find those values of $\phi_{n}$ which minimize (19), subject to the constraint (22).

The phase transition occurs when the curvature of the Gaussian potential for $\phi_{1}$ develops a vanishing moment. This is a first order phase transition 38 . Unlike generic first order phase transitions, it occurs at the point where the curvature of the potential first goes to zero. As a result, there is no energy barrier separating the two phases and no coexistence region of the ordered and disordered phases.

These features of the phase transition are very sensitive to higher order corrections. In the following, we shall take into account the leading effect of higher order corrections.

Since, as the temperature is raised from zero, the variable $\phi_{1}$ is the first to condense, we focus on its effective action. We have computed the relevant parts of the effective action up to three loop order.

First of all, the $\phi_{n}$-independent part of the effective action (what one obtains by putting $\phi_{n}=0$ for $n \neq 0$ ) vanishes up to order $\lambda^{2}$.

The relevant parts which depend on $\phi_{n}$ have the form

$\frac{1}{N^{2}} S_{\text {eff }}=\Delta_{1}(r)\left|\phi_{1}\right|^{2}+\Delta_{2}(r)\left|\phi_{2}\right|^{2}+\lambda P_{1}(r)\left(\phi_{1} \phi_{1} \phi_{-2}+\right.$ c.c. $)+\lambda^{2} P_{2}(r)\left|\phi_{1}\right|^{4}+\ldots$

The coefficients in (23) are

$$
\begin{gathered}
\Delta_{1}(r)=\left[1-8 r^{3}-3 r^{4}-6 r^{2}\right]-24 \lambda\left[\ln (r) r^{2}\left(r^{2}+1\right)(r+1)^{4}\right]- \\
-3 \lambda^{2} r^{2}\left[\begin{array}{r}
\ln (r)^{2}\left(68 r^{10}+352 r^{9}+904 r^{8}+1536 r^{7}+2256 r^{6}+\right. \\
\left.+3104 r^{5}+4120 r^{4}+2304 r^{3}+928 r^{2}+192 r+16\right)- \\
-\ln (r)\left(27 r^{10}+152 r^{9}+390 r^{8}+640 r^{7}+915 r^{6}+1232 r^{5}+\right. \\
\left.\left.+1748 r^{4}+1184 r^{3}+466 r^{2}+440 r+102\right)\right]+\ldots \\
\Delta_{2}(r)=\frac{1}{2}\left(1+8 r^{6}-3 r^{8}-6 r^{4}\right)+\ldots \\
P_{1}(r)=-12 \ln (r) r^{4}\left(2 r^{4}-4 r^{3}+3 r^{2}-4 r+5\right)(r+1)^{4}
\end{array}\right.
\end{gathered}
$$




$$
\begin{array}{r}
P_{2}(r)=3 r^{4}\left[-\ln (r)^{2}\left(136 r^{12}+512 r^{11}+704 r^{10}-1308 r^{8}-1376 r^{7}+1560 r^{6}\right.\right. \\
\left.+6400 r^{5}+10896 r^{4}+8096 r^{3}+2136 r^{2}+1536 r+240\right)+ \\
+\ln (r)\left(27 r^{12}+120 r^{11}+166 r^{10}-32 r^{9}-271 r^{8}-16 r^{7}+1044 r^{6}+\right. \\
\left.\left.+2624 r^{5}+4036 r^{4}+3256 r^{3}+774 r^{2}+768 r+944\right)\right]+\ldots
\end{array}
$$

Eliminating $\phi_{2}$ using its equation of motion, we obtain the effective action for $\phi_{1}$, in the large $N$ limit, and to order $\lambda^{2}$ :

$$
\frac{1}{N^{2}} S_{\mathrm{eff}}=\Delta_{1}(r)\left|\phi_{1}\right|^{2}+\lambda^{2}\left(P_{2}(r)-\frac{\left[P_{1}(r)\right]^{2}}{\Delta_{2}(r)}\right)\left|\phi_{1}\right|^{4}+\ldots
$$

\subsection{Phase transition}

As we raise the temperature from zero, the quadratic term in $\phi_{1}$ in the effective action vanishes at the critical value of $r$,

$$
r_{c}=\frac{1}{3}+\lambda \frac{2^{6} \cdot 5}{3^{5}} \ln (3)-\lambda^{2}\left[\frac{11 \cdot 53 \cdot 3061}{2^{2} \cdot 3^{9}} \ln (3)+\frac{13^{2} \cdot 1867}{2^{4} \cdot 3^{8}} \ln (3)^{2}\right]+\ldots
$$

which translates to the critical temperature

$$
T_{c}=\frac{\mu}{12 \ln (3)}\left[1+\lambda \frac{2^{6} \cdot 5}{3^{4}}-\lambda^{2}\left(\frac{23 \cdot 19927}{2^{2} \cdot 3^{7}}+\frac{1765769}{2^{4} \cdot 3^{8}} \ln (3)\right)+\ldots\right]
$$

The zeroth order term in the critical temperature is the one found in 13 . The term of first order in $\lambda$ agrees with the result quoted in ref. [18].

Also, from (28) we see that the quartic term in $\phi_{1}$ is negative over the entire range $0<r<1$. This means that the phase transition is of first order. When $r$ is just less than the critical $r_{C}=1 / 3$, the extremum of the effective action at $\phi_{1}=0$ is only a local minimum. The effective action has a second zero when

$$
\left|\phi_{1}\right|^{2}=-\frac{1}{\lambda^{2}} \frac{\Delta_{1}(r)}{P_{2}(r)-P_{1}^{2}(r) / \Delta_{2}(r)}
$$

Higher order terms in the effective action are individually small at this value of $\left|\phi_{1}\right|^{2}$ when $-\frac{\Delta_{1}(r)}{P_{2}(r)-P_{1}^{2}(r) / \Delta_{2}(r)}<<1$. We are further constrained by the fact that $\left|\phi_{1}\right| \leq 1$. This requires that $-\frac{\Delta_{1}(r)}{P_{2}(r)-P_{1}^{2}(r) / \Delta_{2}(r)}<\lambda^{2}<<1$. The number $-\frac{\Delta_{1}(r)}{P_{2}(r)-P_{1}^{2}(r) / \Delta_{2}(r)}$ is less than 0.10 in the range $0.2555<r \leq 1 / 3$ and is less than 0.001 in the range $0.3174<r \leq 1 / 3$. 
If $r$ is sufficiently close to $r_{c}$, we can reliably say that the absolute minimum of the potential is not at $\phi_{1}=0$ but is elsewhere. This sets an upper bound on the transition temperature

$$
T_{\text {crit. }}<T_{c}=\frac{\mu}{12 \ln (3)}
$$

The tunnelling barrier for bubble nucleation during the first order phase transition is of order $1 / \lambda^{2}$.

\section{Conclusions}

We have found that the phase transition in the weakly coupled plane wave matrix model is indeed of first order. As the temperature is raised from zero, the curvature contained in the quadratic term in the effective action still vanishes at some critical temperature. However, before that point is reached, when there is still an energy barrier between the two phases, the deconfined phase becomes the lower energy state. This is the generic behavior at a first order phase transition. In fact, this behavior is seen in other adjoint matrix models 41-43. It is also the behavior that is seen in the collapse of Anti de Sitter space to a black hole, which is thought to be the analog of this phase transition in supergravity of a similar deconfinement in $\mathcal{N}=4$ supersymmetric Yang-Mills theory [4].

Our analysis does not allow us to compute the first order phase transition temperature accurately, only to deduce that it is of first order. It does, however, allow us to compute corrections to the Hagedorn temperature. This is the temperature at which, if the confining phase is superheated beyond where it is a global minimum of the free energy, it eventually becomes perturbatively unstable. It is just the place where the corrected curvature of the effective action vanishes.

To conclude, we summarize our results:

- We have computed the full free energy to two loop order: 


$$
\begin{aligned}
& \quad S_{\text {eff }}^{2 \text {-loops }}=-\frac{27 \beta g^{2}}{4 \mu^{2}} \sum_{a b c}\left(-\frac{\left(1-r^{8}\right)}{C_{a b}^{\omega_{1}} C_{c a}^{\omega_{1}}}-20 \frac{\left(1-r^{4}\right)}{C_{a b}^{\omega_{2}} C_{c a}^{\omega_{2}}}-12 \frac{\left(1-r^{8}\right)\left(1-r^{4}\right)}{C_{a b}^{\omega_{1}} C_{c a}^{\omega_{2}}}\right. \\
& +\frac{\left(r^{8}+4 r^{4}+1\right)\left(r^{4}-1\right)^{4}+\left[\cos \beta A_{a b}+\cos \beta A_{b c}+\cos \beta A_{c a}\right] 2 r^{4}\left(r^{4}-1\right)^{4}}{C_{a b}^{\omega_{1}} C_{b c}^{\omega_{1}} C_{c a}^{\omega_{1}}} \\
& +16 \frac{r^{3}\left(r^{4}-r^{2}+1\right)\left(r^{4}-1\right)^{2}\left(r^{2}+1\right)\left[\cos \beta A_{a b}+\cos \beta A_{b c}\right]+r^{6}\left(r^{4}-1\right)^{2}\left[2+2 \cos \beta A_{c a}\right]}{\bar{C}_{a b} \bar{C}_{b c} C_{c a}^{\omega_{1}}} \\
& \left.+32 \frac{r^{3}\left(r^{4}-1\right)^{2}\left(r^{2}+1\right)\left[\cos \beta A_{a b}+\cos \beta A_{b c}\right]+r^{2}\left(r^{8}-1\right)\left(r^{4}-1\right) \cos \beta A_{c a}+\left(r^{4}-1\right)^{2}\left(r^{8}+1\right)}{\bar{C}_{a b} \bar{b}_{b c} C_{c a}^{\omega_{2}}}\right)
\end{aligned}
$$

Where we have:

$$
\begin{aligned}
& C_{a b}^{\omega_{1}}=1-2 r^{4} \cos \beta A_{a b}+r^{8} \\
& C_{a b}^{\omega_{2}}=1-2 r^{2} \cos \beta A_{a b}+r^{4} \\
& \bar{C}_{a b}=1+2 r^{3} \cos \beta A_{a b}+r^{6}
\end{aligned}
$$

This expression can be restated to exhibit its $\phi_{n}$ dependence:

$$
\begin{aligned}
\frac{1}{N^{2}} S_{\text {eff }}^{2 \text {-loops }} & =3 \lambda \ln (r) \sum_{m n} \phi_{n} \phi_{m} \phi_{-m-n}\left[-4 \frac{r^{8}-r^{4}+1}{\left(r^{4}+1\right)^{2}} r^{4|n|+4|m|}\right. \\
& +16 \frac{\left(r^{4}-r^{2}+1\right)\left(r^{2}+1\right)^{2}}{\left(r^{4}+r^{2}+1\right)\left(r^{4}+1\right)}(-1)^{n} r^{3|n|+4|m|}-16 \frac{\left(r^{2}+1\right)^{2}}{r^{4}+r^{2}+1}(-1)^{n+m} r^{3|n|+3|m|} \\
& +32 \frac{\left(r^{2}+1\right)^{2}}{r^{4}+r^{2}+1}(-1)^{n} r^{3|n|+2|m|}-12 r^{4|n|+2|m|}-20 r^{2|n|+2|m|} \\
& -4 \frac{\left(r^{4}-1\right)\left(r^{8}+r^{4}+1\right)}{\left(r^{4}+1\right)^{3}} F_{m n}(4,4)+16 \frac{\left(r^{2}+1\right)\left(r^{10}-1\right)}{\left(r^{4}+1\right)\left(r^{4}+r^{2}+1\right)^{2}}(-1)^{m} F_{m n}(3,4) \\
& \left.-16 \frac{\left(r^{2}+1\right)\left(r^{6}-r^{4}+r^{2}-1\right)}{\left(r^{4}+r^{2}+1\right)^{2}}(-1)^{m} F_{m n}(3,2)\right]
\end{aligned}
$$

Where we define the function $F_{m n}(a, b)$ in the following manner:

$$
F_{m n}(a, b)= \begin{cases}F_{m n}^{1}(a, b) & m, n \geq 0 \quad \text { or } \quad m, n<0 \\ F_{m n}^{2}(a, b) & n<0, m \geq-n \quad \text { or } n \geq 0, m<-n \\ F_{m n}^{3}(a, b) & m<0, m \geq-n \text { or } m \geq 0, m<-n\end{cases}
$$


And where we have:

$$
\begin{aligned}
F_{m n}^{1}(a, b)=r^{a(2+n+m)+b} & {\left[\frac{r^{b(n+m)-a n}}{1-r^{2 a+b}}+\frac{r^{-b-2 a+a n}}{1-r^{2 a+b}}+\frac{r^{-2 a-a n+b(n+m)}}{r^{b}-1}\right.} \\
& \left.-\frac{r^{-2 a-a n+b n}}{r^{b}-1}-\frac{r^{-2 a-a n+b n}}{-r^{b}+r^{2 a}}+\frac{r^{-2 a+a n}}{-r^{b}+r^{2 a}}\right]
\end{aligned}
$$

$$
\begin{aligned}
F_{m n}^{2}(a, b)=r^{a(n+m)+b} & {\left[\frac{r^{2 a-a n+b(n+m)}}{1-r^{2 a+b}}+\frac{r^{-b-a n-b n}}{1-r^{2 a+b}}\right.} \\
+ & \left.\frac{r^{-a n+b(n+m)}+r^{-b-a n}-r^{-a n}}{r^{b}-1}-\frac{r^{-b n-b-a n}}{1-r^{b}}\right]
\end{aligned}
$$

$$
\begin{aligned}
F_{m n}^{3}(a, b)=r^{a(n-m)+b} & {\left[\frac{r^{-a n+2 a+b n}}{1-r^{2 a+b}}+\frac{r^{-b+a n+2 a m}}{1-r^{2 a+b}}+\frac{r^{-a n+b n}}{r^{b}-1}\right.} \\
& \left.-\frac{r^{-a n+b(n+m)}}{r^{b}-1}-\frac{r^{-a n+b(n+m)}}{-r^{b}+r^{2 a}}+\frac{r^{a n+2 a m}}{-r^{b}+r^{2 a}}\right]
\end{aligned}
$$

- In the zero temperature limit, $r \rightarrow 0$. We then see that the free energy is $1+20+12-1-32=0$, which is what is expected from supersymmetry.

- We obtain parts of the free energy to three loop order

- We check our three-loop computation by taking the zero temperature limit of it and finding that the free energy vanishes as the temperature is taken to zero. This is expected as a result of the supersymmetry of the model which is restored at zero temperature.

- We also find that the $\phi_{n}$-independent part of the free energy vanishes to this order. Note that this is not the same as the zero temperature limit.

- We find the full shift in $T_{c}$ to order $\lambda^{2}$. It is given in eqn. (30).

- The form of the effective action that we find confirms the first order nature of the phase transition at weak coupling. 


\section{Acknowledgments}

This work was partially supported by NSERC of Canada, the String Theory Collaborative Research Group of the Pacific Institute for Mathematical Sciences and the Strings and Particles Collaborative Research Team of the Pacific Institute for Theoretical Physics.

\section{Appendix A: Three loops}

To do perturbation theory, we split the Euclidean action into three parts, a free action, an interaction action with three-point vertices and an interaction action with four-point vertices,

$$
\begin{array}{r}
S_{0}=\int_{-\beta / 2}^{\beta / 2} d \tau \operatorname{Tr}\left(\frac{1}{2} X^{i}\left(-D^{2}+(\mu / 6)^{2}\right) X^{i}+\frac{1}{2} X^{\bar{a}}\left(-D^{2}+(\mu / 3)^{2}\right) X^{\bar{a}}+\right. \\
\left.+\psi^{\dagger I \alpha}(-D+\mu / 4) \psi_{I \alpha}\right) \\
S_{3}=\int_{-\beta / 2}^{\beta / 2} d \tau R^{\frac{3}{2}} \operatorname{Tr}\left(i \mu \frac{1}{3} \epsilon_{\bar{a} \bar{b} \bar{c}} X^{\bar{a}} X^{\bar{b}} X^{\bar{c}}+\psi^{\dagger I \alpha} \sigma_{\alpha}^{\bar{a} \beta}\left[X^{\bar{a}}, \psi_{I \beta}\right]-\right. \\
\left.-\frac{1}{2} \epsilon_{\alpha \beta} \psi^{\dagger \alpha I} \mathrm{~g}_{I J}^{i}\left[X^{i}, \psi^{\dagger \beta J}\right]+\frac{1}{2} \epsilon^{\alpha \beta} \psi_{\alpha I}\left(\mathrm{~g}^{i \dagger}\right)^{I J}\left[X^{i}, \psi_{\alpha J}\right]\right) \\
S_{4}=-\int_{-\beta / 2}^{\beta / 2} d \tau \frac{R^{3}}{4} \operatorname{Tr}\left(\left[X^{i}, X^{j}\right]^{2}+\left[X^{\bar{a}}, X^{\bar{b}}\right]^{2}+2\left[X^{\bar{a}}, X^{i}\right]^{2}\right)
\end{array}
$$

respectively.

The effective action $S_{\text {eff }}[A]$ is obtained by a perturbative expansion of the partition function.

$$
e^{-S_{\mathrm{eff}}[A]}=\int[d X][d \psi] e^{-S_{0}-S_{3}-S_{4}} \quad, \quad S_{\mathrm{eff}}[A]=1-<e^{-S_{3}-S_{4}}>
$$

where the bracket indicates the connected part of the expectation value in the free theory. Taylor expanding the exponential of interactions and retaining those terms which have non-zero contributions to two loop order gives

$$
\begin{aligned}
S_{\text {eff }}= & \sum_{a \neq b} \ln \left[\frac{\operatorname{det}^{3 / 2}\left(-D_{a b}^{2}+\frac{\mu^{2}}{9}\right) \operatorname{det}^{3}\left(-D_{a b}^{2}+\frac{\mu^{2}}{36}\right)}{\operatorname{det}\left(-D_{a b}\right) \operatorname{det}^{8}\left(-D_{a b}+\frac{\mu}{4}\right)}\right]+ \\
& +\left(<S_{4}>-\frac{1}{2}<S_{3}^{2}>\right)-\left(\frac{1}{2}<S_{4}^{2}>-\frac{1}{2}<S_{3}^{2} S_{4}>+\frac{1}{24}<S_{3}^{4}>\right)+\ldots(
\end{aligned}
$$


The first line on the right-hand-side is one-loop, the bracket after it contains two-loop and the next bracket the three-loop contributions.

The appropriate two-loop Feynman diagrams are as follows, where a dotted line represents a fermion, and a solid line either flavour of scalar.
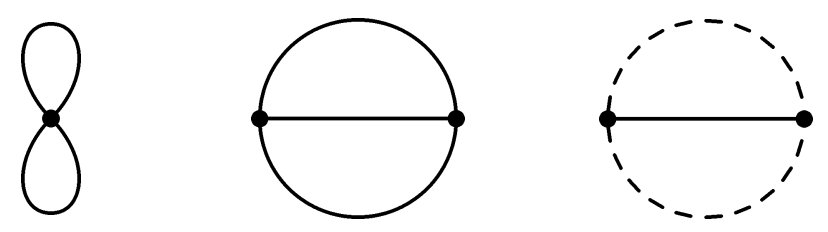

At three loops there are many more diagrams. The following figures show the three loop diagrams and their associated zero temperature limits. The letter "P" denotes the circulation of the $m=\mu / 3$ scalar, while "Q" denotes the $m=\mu / 6$ scalar. Where there is no indication, it is obvious from the allowed interactions dictated by the action.

\section{Cat's Eye Diagram}

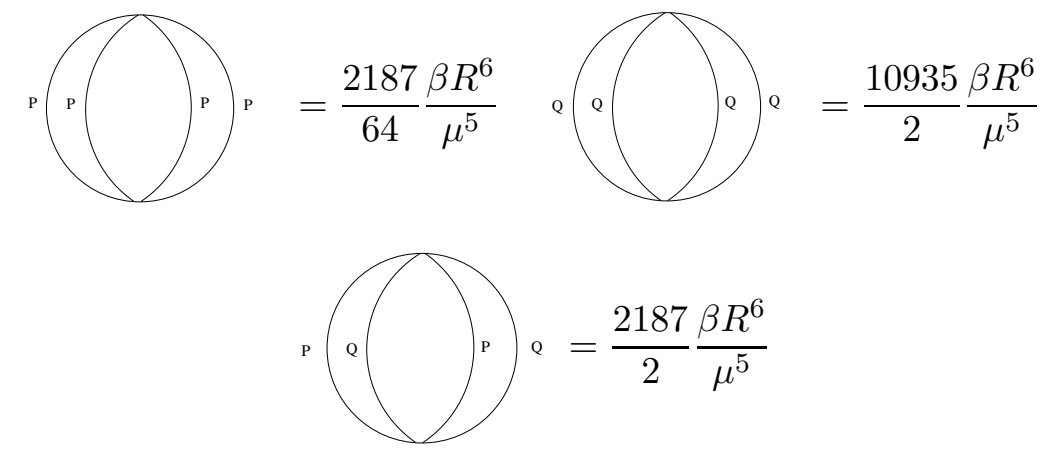

\section{Triple Bubble Diagram}

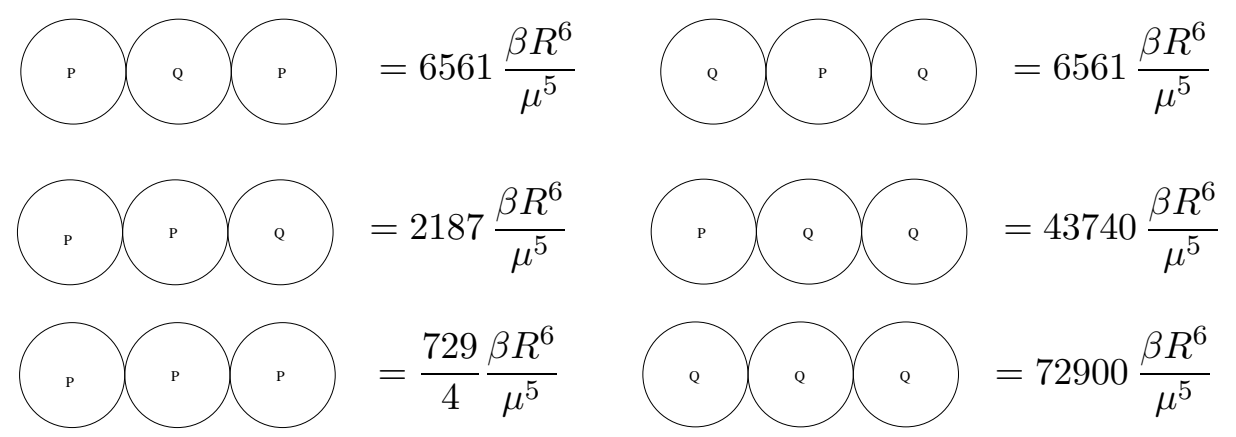




\section{Theta-Bubble Diagram}

$$
\begin{aligned}
& \bigcirc=-\frac{10935}{32} \frac{\beta R^{6}}{\mu^{5}} \bigcirc=-729 \frac{\beta R^{6}}{\mu^{5}} \\
& \therefore=0 \quad \square=-4374 \frac{\beta R^{6}}{\mu^{5}}
\end{aligned}
$$

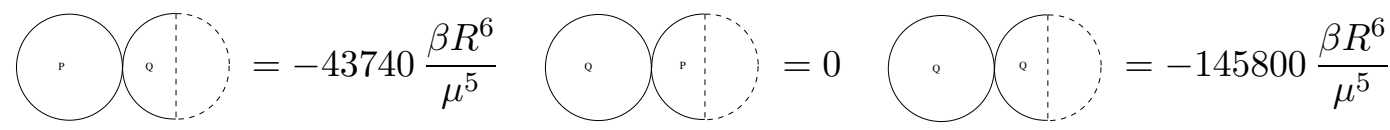

\section{Circle-T Diagram}

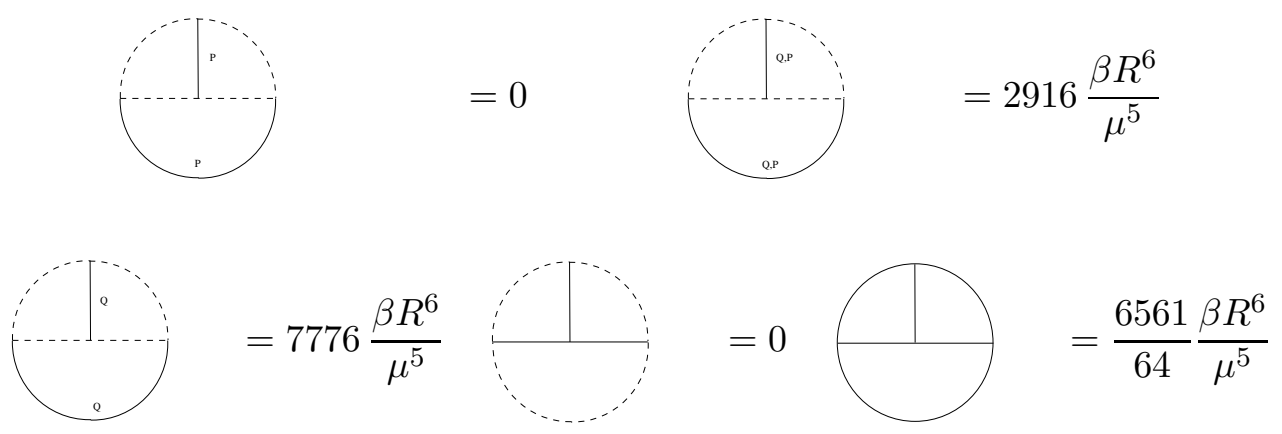

\section{Two-Rung Ladder Diagrams}

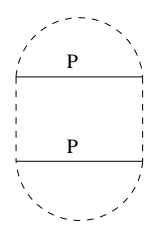

$$
=0
$$

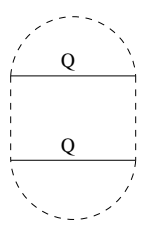

$$
=-34992 \frac{\beta R^{6}}{\mu^{5}}
$$

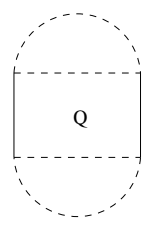

$$
=73872 \frac{\beta R^{6}}{\mu^{5}}
$$

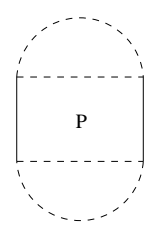

$$
=0
$$

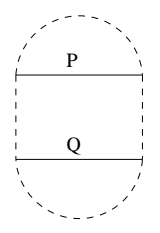

$$
=5832 \frac{\beta R^{6}}{\mu^{5}}
$$
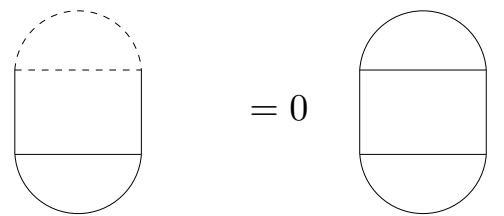

$$
=\frac{24057}{32} \frac{\beta R^{6}}{\mu^{5}}
$$


One can check that the sum of the above factors is zero, as garaunteed by SUSY.

The vertices for these diagrams can be deduced from the interaction terms in the action. The propagators depend on temperature and are discussed in the appendix below.

The technique that we use is to work in time, rather than momentum representation of the propagators. The integration over times is elementary and most of the work involves extracting the moments in $\eta_{a} \equiv e^{i \beta A_{a}}$. Liberal use of computer algebra systems was used to unravel the expansion of diagrams in this object.

\section{Appendix B: Thermal Green functions}

The free field correlation function of the scalar field is

$$
\left\langle X_{a b}^{i}(\tau) X_{c d}^{j}(0)\right\rangle=\delta^{i j} \delta_{a d} \delta_{b c}\left[g_{1}(\tau)\right]_{a b}
$$

where the Green function is

$$
\begin{aligned}
{\left[g_{1}(\tau)\right]_{a b}=(} & \left.\frac{1}{-D_{a b}^{2}+(\mu / 6)^{2}} \mid 0\right) \\
=-\frac{\beta \eta^{-\tau / \beta}}{4 \ln r}[ & {\left[\frac{1}{1-\frac{1}{\eta} r^{2}}\left[r^{2}\right]^{\tau / \beta}+\frac{\eta r^{2}}{1-\eta r^{2}}\left[r^{2}\right]^{-\tau / \beta}\right) \theta(\tau)+} \\
& \left.\quad+\left(\frac{\frac{1}{\eta} r^{2}}{1-\frac{1}{\eta} r^{2}}\left[r^{2}\right]^{\tau / \beta}+\frac{1}{1-\eta r^{2}}\left[r^{2}\right]^{-\tau / \beta}\right) \theta(-\tau)\right]
\end{aligned}
$$

where $\eta=e^{i \beta A_{a b}}=\eta_{a} / \eta_{b}$ and $r=e^{-\beta \mu / 12}$. This Green function is defined on the interval $\tau / \beta \in(-1 / 2,1 / 2)$, with $g_{1}(1 / 2)=g_{1}(-1 / 2)$ and it must be extended periodically to all real values $\tau / \beta+$ integers. An alternative expression which is sometimes useful is

$$
g_{1}(\tau)=-\frac{\beta \eta^{-\tau / \beta}}{4 \ln r}\left(\left[r^{2}\right]^{|\tau| / \beta}+\sum_{n=1}^{\infty} r^{2 n}\left[\eta^{-n}\left[r^{2}\right]^{\tau / \beta}+\eta^{n}\left[r^{2}\right]^{-\tau / \beta}\right]\right)
$$

The first term is the zero temperature Euclidean green function and the last two terms are the homogeneous solutions of the Euclidean wave equation which must be added to the first term in order to satisfy the periodic boundary conditions. Note that both green functions have a coefficient $\eta^{-\tau / \beta}$. This factor cancels identically in all vacuum diagrams. It has been separated explicitly since some loop integrations are easier once it is canceled.

Similarly,

$$
\left\langle X_{a b}^{\bar{a}}(\tau) X_{c d}^{\bar{b}}(0)\right\rangle_{0}=\delta^{\bar{a} \bar{b}} \delta_{a d} \delta_{b c}\left[g_{2}(\tau)\right]_{a b}
$$




$$
\begin{aligned}
{\left[g_{2}(\tau)\right]_{a b}=} & \left(\tau\left|\frac{1}{-D_{a b}^{2}+(\mu / 3)^{2}}\right| 0\right) \\
= & -\frac{\beta \eta^{-\tau / \beta}}{8 \ln r}\left[\left(\frac{1}{1-\frac{1}{\eta} r^{4}}\left[r^{4}\right]^{\tau / \beta}+\frac{\eta r^{4}}{1-\eta r^{4}}\left[r^{4}\right]^{-\tau / \beta}\right) \theta(\tau)+\right. \\
& \left.\quad+\left(\frac{\frac{1}{\eta} r^{4}}{1-\frac{1}{\eta} r^{4}}\left[r^{4}\right]^{\tau / \beta}+\frac{1}{1-\eta r^{4}}\left[r^{4}\right]^{-\tau / \beta}\right) \theta(-\tau)\right] \\
= & -\frac{\beta \eta^{-\tau / \beta}}{8 \ln r}\left(\left[r^{4}\right]^{|\tau| / \beta}+\sum_{n=1}^{\infty} r^{4 n}\left[\eta^{-n}\left[r^{4}\right]^{\tau / \beta}+\eta^{n}\left[r^{4}\right]^{-\tau / \beta}\right]\right)
\end{aligned}
$$

The Fermion Green function is

$$
\left[g_{f}(\tau)\right]_{a b}=\left(\tau\left|\frac{1}{-D_{a b}+(\mu / 4)}\right| 0\right)=-\eta^{-\tau / \beta} \frac{\left[r^{3}\right]^{\tau / \beta}}{1+r^{3} / \eta}\left(\theta(\tau)-\frac{1}{\eta} r^{3} \theta(-\tau)\right)
$$

It is antiperiodic $g_{f}(-1 / 2)=-g_{f}(1 / 2)$.

Note that the expressions in (42), (43) are unchanged by the replacement $r \rightarrow 1 / r$.

\section{References}

[1] T. Banks, W. Fischler, S. H. Shenker and L. Susskind, "M theory as a matrix model: A conjecture," Phys. Rev. D 55, 5112 (1997) arXiv:hep-th/9610043.

[2] D. Berenstein, J. M. Maldacena and H. Nastase, "Strings in flat space and pp waves from $\mathrm{N}=4$ super Yang Mills," JHEP 0204, 013 (2002) arXiv:hep-th/0202021.

[3] K. Dasgupta, M. M. Sheikh-Jabbari and M. Van Raamsdonk, "Matrix perturbation theory for M-theory on a PP-wave," JHEP 0205, 056 (2002) arXiv:hep-th/0205185.

[4] N. w. Kim and J. Plefka, "On the spectrum of pp-wave matrix theory," Nucl. Phys. B 643, 31 (2002) arXiv:hep-th/0207034.

[5] K. Dasgupta, M. M. Sheikh-Jabbari and M. Van Raamsdonk, 'Protected multiplets of M-theory on a plane wave," JHEP 0209, 021 (2002) arXiv:hep-th/0207050.

[6] N. Kim and J. H. Park, "Superalgebra for M-theory on a pp-wave," Phys. Rev. D 66, 106007 (2002) arXiv:hep-th/0207061.

[7] J. H. Park, "Supersymmetric objects in the M-theory on a pp-wave," JHEP 0210, 032 (2002) arXiv:hep-th/0208161. 
[8] J. Maldacena, M. M. Sheikh-Jabbari and M. Van Raamsdonk, "Transverse fivebranes in matrix theory," JHEP 0301, 038 (2003) arXiv:hep-th/0211139.

[9] N. w. Kim, T. Klose and J. Plefka, "Plane-wave matrix theory from N = 4 super Yang-Mills on R x $\mathrm{S}^{* *} 3$," Nucl. Phys. B 671, 359 (2003) arXiv:hep-th/0306054.

[10] S. R. Das, J. Michelson and A. D. Shapere, "Fuzzy spheres in pp-wave matrix string theory," arXiv:hep-th/0306270.

[11] T. Klose and J. Plefka, "On the integrability of large N plane-wave matrix theory," Nucl. Phys. B 679, 127 (2004) arXiv:hep-th/0310232.

[12] G. 't Hooft, "A Planar Diagram Theory For Strong Interactions," Nucl. Phys. B 72, 461 (1974).

[13] K. Furuuchi, E. Schreiber and G. W. Semenoff, "Five-brane thermodynamics from the matrix model," arXiv:hep-th/0310286.

[14] J. T. Yee and P. Yi, "Instantons of M(atrix) theory in pp-wave background," JHEP 0302, 040 (2003) arXiv:hep-th/0301120.

[15] H. j. Shin and K. Yoshida, "Thermodynamics of fuzzy spheres in pp-wave matrix model," arXiv:hep-th/0401014.

[16] H. Shin and K. Yoshida, "Membrane fuzzy sphere dynamics in plane-wave matrix model," arXiv:hep-th/0409045.

[17] G. W. Semenoff, "Matrix model thermodynamics," arXiv:hep-th/0405107.

[18] M. Spradlin, M. Van Raamsdonk and A. Volovich, "Two-loop partition function in the planar plane-wave matrix model," arXiv:hep-th/0409178.

[19] A. M. Polyakov, "Thermal Properties Of Gauge Fields And Quark Liberation," Phys. Lett. B 72, 477 (1978).

[20] L. Susskind, "Lattice Models Of Quark Confinement At High Temperature," Phys. Rev. D 20 (1979) 2610.

[21] L. D. McLerran and B. Svetitsky, "A Monte Carlo Study Of SU(2) YangMills Theory At Finite Temperature," Phys. Lett. B 98, 195 (1981).

[22] L. G. Yaffe and B. Svetitsky, "First Order Phase Transition In The SU(3) Gauge Theory At Finite Temperature," Phys. Rev. D 26, 963 (1982).

[23] N. Weiss, "The Effective Potential For The Order Parameter Of Gauge Theories At Finite Phys. Rev. D 24, 475 (1981).

[24] B. Svetitsky and L. G. Yaffe, "Critical Behavior At Finite Temperature Confinement Transitions," Nucl. Phys. B 210, 423 (1982). 
[25] N. Weiss, "The Wilson Line In Finite Temperature Gauge Theories," Phys. Rev. D 25, 2667 (1982).

[26] G. Grignani, G. W. Semenoff and P. Sodano, "Confinement - deconfinement transition in three-dimensional QED," Phys. Rev. D 53, 7157 (1996) arXiv:hep-th/9504105.

[27] G. Grignani, G. W. Semenoff, P. Sodano and O. Tirkkonen, "Charge Screening in the Finite Temperature Schwinger Model," Int. J. Mod. Phys. A 11, 4103 (1996) arXiv:hep-th/9511110.

[28] G. Grignani, G. W. Semenoff, P. Sodano and O. Tirkkonen, "Charge Screening and Confinement in the Hot 3D-QED," Nucl. Phys. B 473, 143 (1996) arXiv:hep-th/9512048.

[29] G. Grignani, L. Paniak, G. W. Semenoff and P. Sodano, "Loop correlators and theta states in two-dimensional Yang-Mills theory," Annals Phys. 260, 275 (1997) arXiv:hep-th/9705102.

30.

[30] A. Dumitru, Y. Hatta, J. Lenaghan, K. Orginos and R. D. Pisarski, "Deconfining phase transition as a matrix model of renormalized Polyakov loops," Phys. Rev. D 70, 034511 (2004) arXiv:hep-th/0311223.

[31] B. Sundborg, "The Hagedorn transition, deconfinement and N = 4 SYM theory," Nucl. Phys. B 573, 349 (2000) arXiv:hep-th/9908001.

[32] A. M. Polyakov, "Gauge fields and space-time," Int. J. Mod. Phys. A 17S1, 119 (2002) arXiv:hep-th/0110196.

[33] O. Aharony, J. Marsano, S. Minwalla, K. Papadodimas and M. Van Raamsdonk, "The Hagedorn / deconfinement phase transition in weakly coupled large N gauge theories," arXiv:hep-th/0310285.

[34] H. J. Schnitzer, "Confinement / deconfinement transition of large N gauge theories with N(f) Nucl. Phys. B 695, 267 (2004) arXiv:hep-th/0402219.

[35] H. Liu, "Fine structure of Hagedorn transitions," arXiv:hep-th/0408001

[36] M. Spradlin and A. Volovich, "A pendant for Polya: The one-loop partition function of $\mathrm{N}=4 \mathrm{SYM}$ on $\mathrm{R} \times \mathrm{S}(3)$," arXiv:hep-th/0408178.

[37] D. J. Gross and E. Witten, "Possible Third Order Phase Transition In The Large N Lattice Gauge Theory," Phys. Rev. D 21, 446 (1980).

[38] For a review, particularly with a discussion of the phase transition in adjoint matrix models, see G. W. Semenoff and R. J. Szabo, "Fermionic Matrix Models," Int. J. Mod. Phys. A 12, 2135 (1997) arXiv:hep-th/9605140. 
[39] J. Ambjorn, Y. M. Makeenko and G. W. Semenoff, "Thermodynamics of D0-branes in matrix theory," Phys. Lett. B 445, 307 (1999) arXiv:hep-th/9810170.

[40] Y. Makeenko, "Formulation of matrix theory at finite temperature," Fortsch. Phys. 48, 171 (2000) arXiv:hep-th/9903030.

[41] G. W. Semenoff, O. Tirkkonen and K. Zarembo, "Exact solution of the one-dimensional non-Abelian Coulomb gas at large N," Phys. Rev. Lett. 77, 2174 (1996) arXiv:hep-th/9605172.

[42] G. W. Semenoff and K. Zarembo, "Adjoint non-Abelian Coulomb gas at large N," Nucl. Phys. B 480, 317 (1996) arXiv:hep-th/9606117.

[43] C. R. Gattringer, L. D. Paniak and G. W. Semenoff, "Deconfinement transition for quarks on a line," Annals Phys. 256, 74 (1997) arXiv:hep-th/9612030.

[44] E. Witten, "Anti-de Sitter space, thermal phase transition, and confinement in gauge theories," Adv. Theor. Math. Phys. 2, 505 (1998) arXiv:hep-th/9803131. 